\title{
Linear and quadratic magneto-optical Kerr effects in continuous and granular ultrathin monocrystalline Fe films
}

\author{
B. Sepúlveda, ${ }^{*}$ Y. Huttel, C. Martínez Boubeta, A. Cebollada, and G. Armelles \\ Instituto de Microelectrónica de Madrid-IMM (CNM-CSIC), Isaac Newton 8 (PTM) Tres Cantos, Madrid 28760, Spain
}

(Received 15 October 2002; revised manuscript received 11 April 2003; published 1 August 2003)

\begin{abstract}
The effects of nanostructuration on the magneto-optic properties of ultrathin monocrystalline iron films grown on $\mathrm{MgO}$ (001) are investigated through the magneto-optic coefficients, which have a linear and quadratic dependence on the magnetization. The photon energy dependence of such magneto-optic coefficients is determined by measuring the relative variations of the reflectivity $\left(\Delta R_{p p} / R_{p p}\right)$ for $p$-polarized incident and reflected light ( $p-p$ polarized) when the magnetization rotates in the plane of the sample. Thick Fe films present a magneto-optical anisotropy, which has a quadratic dependence on the magnetization. Such anisotropy is strongly reduced in nanostructured iron thin films formed by nanometric iron islands. The modifications induced by the nanostructuration are stronger for the magneto-optical coefficients, which have a quadratic dependence on the magnetization in contrast to the linear terms. A self-consistent effective-medium formalism is presented that explains the modifications induced in the magneto-optical coefficients by nanostructuration.
\end{abstract}

DOI: $10.1103 / P h y s R e v B .68 .064401$

PACS number(s): 78.20.Ls, 75.75.+a, 78.20.-e, 78.20.Ci

\section{INTRODUCTION}

Nowadays devices such as sensors, read heads, or communication aids tend toward reducing their dimensions to increase their performance. Such reduction of the physical dimensions is likely to modify the electronic structure of the materials, and consequently, to change their optical properties. In the case of ferromagnetic ultrathin layers, photoemission $^{1-4}$ and magneto-optic studies ${ }^{5-8}$ have been employed to observe the quantum confinement effects. The later studies are based on Kerr effects ${ }^{5-7}$ or magnetic secondharmonic generation. ${ }^{8}$ The Kerr effect studies provide information on electronic structure through off-diagonal elements of the dielectric tensor. Such measurements have allowed the detection of oscillations in the Kerr signal as a function of the film thickness for ultrathin iron films due to the presence of quantum well states. ${ }^{5-7}$ Nevertheless the magneto-optical properties of ultrathin magnetic layers may also be affected by the growth mode, ${ }^{9-14}$ and the assignment of changes in the magneto-optical spectra of ultrathin films to changes in the electronic structure of the layers is not always evident. Spin-orbit interaction is the origin of magneto-optical effects, and clear relations have been established between magnetooptical activity and other spin-orbit-related effects such as magnetic anisotropy. For example, polar Kerr rotation studies have evidenced the magneto-optical anisotropy in hcp monocrystalline Co films and their relation with magnetocrystalline anisotropy. ${ }^{15}$ However, the magneto-optical anisotropy observed in this system is due to the hexagonal structure of Co and has a linear dependence on the magnetization. Such a type of magneto-optical anisotropy was not observed in fcc Co and will not be present in other cubic systems such as bcc iron. Nevertheless, these crystallographic systems may present another type of magneto-optical anisotropy, having a quadratic dependence on the magnetization, purely induced by the spin-orbit interaction. ${ }^{16}$ This anisotropy may be affected by the reduction of the size of the structures and, also, by the morphology of the films. The present study is mainly focused on the magneto-optic effects of nanostructuration in ultrathin monocrystalline $\mathrm{Fe}$ films grown on $\mathrm{MgO}$ (001). We show that changes of the morphology of the layers have drastic effects on the magneto-optical properties, as was previously pointed out in specific cases by Menéndez et al., ${ }^{17}$ and which can be understood with the self-consistent effective-medium formalism presented here.

The paper is organized as follows: the theoretical formalism is presented in Sec. II and the experimental details in Sec. III. Section IV is devoted to the experimental results and simulations and the corresponding discussion is given in Sec. V. The last section presents the conclusions.

\section{THEORETICAL FRAMEWORK}

The magnetization dependence of the different elements of the dielectric tensor can be described, up to second-order terms in magnetization, as follows: ${ }^{18}$

$$
\varepsilon_{i j}=\varepsilon_{i j 0}+\sum_{k} a_{i j k} M_{k}+\sum_{k l} b_{i j k l} M_{k} M_{l}+\ldots
$$

where $\varepsilon_{i j 0}$ are the dielectric tensor elements with zero magnetization, $M_{i}=m_{i} / M_{s}$, with $m_{i}$ being the $i$ component of the magnetization and $M_{s}$ the saturation magnetization. First-order dependence is described by a third rank tensor $a_{i j k}$, and the second-order dependence is described by a fourth rank tensor $b_{i j k l}$. For magnetic materials with cubic symmetry, such as iron, there is only one independent linear magneto-optic coefficient in $a_{i j k},{ }^{18}$ which we call $a$, while three magneto-optic quadratic coefficients (among 81) remain independent in $b_{i j k l}\left(b_{11}, b_{12}\right.$, and $b_{44}$ in contracted notation), ${ }^{18,19}$ and $\varepsilon_{x x 0}=\varepsilon_{y y 0}=\varepsilon_{z z 0}$. In this expansion, all the magneto-optic coefficients are wavelength dependent. Due to symmetry considerations off-diagonal elements of the dielectric tensor have a linear and quadratic dependence on 
magnetization, while the dependence is only quadratic for diagonal terms.

To show the optical anisotropy induced by quadratic magneto-optic coefficients we first consider a cubic magnetic medium, magnetized in the $x$ direction, in two different situations: one with the reference system parallel to crystallographic axes $\{[100],[010],[001]\}$, which is described by the following dielectric tensor:

$$
\varepsilon=\left(\begin{array}{ccc}
\varepsilon_{x x 0}+b_{11} & 0 & 0 \\
0 & \varepsilon_{x x 0}+b_{12} & -a \\
0 & a & \varepsilon_{x x 0}+b_{12}
\end{array}\right),
$$

and another with the reference system parallel to $\{[110],[1-10],[001]\}$ directions, whose dielectric tensor is expressed by

$$
\varepsilon=\left(\begin{array}{ccc}
\varepsilon_{x x 0}+\frac{b_{11}+b_{12}}{2}+b_{44} & 0 & 0 \\
0 & \varepsilon_{x x 0}+\frac{b_{11}+b_{12}}{2}-b_{44} & -a \\
0 & a & \varepsilon_{x x 0}+b_{12}
\end{array}\right)
$$

As a consequence, a plane wave propagating in the $z$ direction, with the electric field perpendicular to the magnetization $(y$ direction), will have a different spatial dependence (different refractive index) in each crystallographic orientation, unless

$$
b_{44}=\frac{1}{2}\left(b_{11}-b_{12}\right) .
$$

In this situation the magnetic material is isotropic ${ }^{19}$ and the magnetization-induced optical anisotropy disappears.

In the present study the magnetization is rotating in the plane of the sample, i.e., the $X Y$ plane. In the case of a cubic iron, if the reference system $\{x, y, z\}$ is parallel to the main crystallographic directions $\{[100],[010],[001]\}$, the dielectric tensor becomes

$$
\varepsilon(\bar{M})=\left[\begin{array}{ccc}
\varepsilon_{x x 0}+b_{11} M_{x}^{2}+b_{12} M_{y}^{2} & 2 b_{44} M_{x} M_{y} & a M_{y} \\
2 b_{44} M_{x} M_{y} & \varepsilon_{x x 0}+b_{12} M_{x}^{2}+b_{11} M_{y}^{2} & -a M_{x} \\
-a M_{y} & a M_{x} & \varepsilon_{x x 0}+b_{12}\left(M_{x}^{2}+M_{y}^{2}\right)
\end{array}\right] .
$$

On the other hand, if the reference system $\{x, y, z\}$ is parallel to crystallographic directions $\{[110],[-110],[001]\}$, the dielectric tensor has the following form:

$\varepsilon(\bar{M})$

$$
=\left[\begin{array}{ccc}
\varepsilon_{x x 0}+\frac{b_{11}+b_{12}}{2}\left(M_{x}^{2}+M_{y}^{2}\right)+b_{44}\left(M_{x}^{2}-M_{y}^{2}\right) & -\frac{b_{11}-b_{12}}{2} M_{x} M_{y} & a M_{y} \\
-\frac{b_{11}-b_{12}}{2} M_{x} M & \varepsilon_{x x 0}+\frac{b_{11}+b_{12}}{2}\left(M_{x}^{2}+M_{y}^{2}\right)-b_{44}\left(M_{x}^{2}-M_{y}^{2}\right) & -a M_{x} \\
-a M_{y} & a M_{x} & \varepsilon_{x x 0}+b_{12}\left(M_{x}^{2}+M_{y}^{2}\right)
\end{array}\right] .
$$

As can be seen by comparing Eqs. (5) and (6), a rotation of $45^{\circ}$ around the $z$ axis does not modify the linear magnetization dependence in off-diagonal elements. In contrast, the quadratic magnetization dependence changes in both diagonal and off-diagonal elements, resulting as well in a magnetization-induced optical anisotropy due to quadratic magneto-optic coefficients.
To determine the different magneto-optic terms of the dielectric tensor we have measured the relative variations of the reflectivity $\left(\Delta R_{p p} / R_{p p}\right)$ for $p$-polarized incident and reflected light ( $p-p$ polarized) when the magnetization rotates in the $x y$ plane at a frequency $\omega$ (see Fig. 1). In this configuration $\mathbf{M}=M(\cos \omega t, \sin \omega t, 0)$ where $M$ is the sample magnetization and 


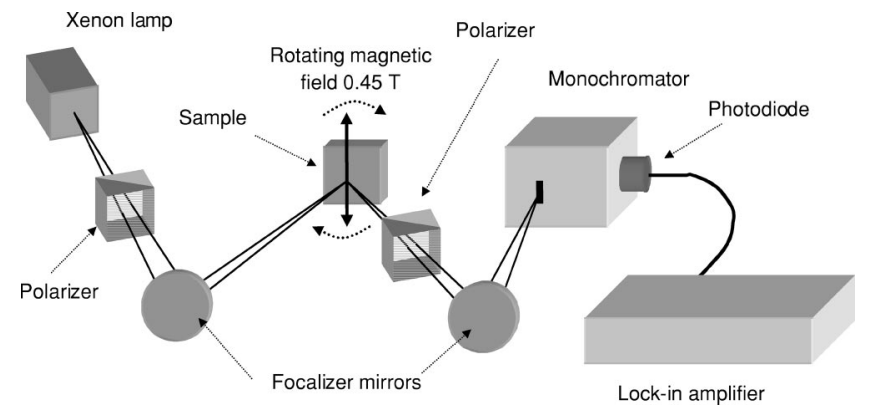

FIG. 1. Diagram of the magneto-optical experimental setup used for the present study.

$$
\frac{\Delta R_{p p}}{R_{p p}}=\frac{R_{p p}(M)-R_{p p}(0)}{R_{p p}(0)} .
$$

The harmonic decomposition of the magnetization can be introduced since the magnetic field used in the present study is much higher than the saturation field of the samples.

In those measurements different magneto-optical coefficients are involved. In order to prove such an assumption, for simplicity, we examine the variations of reflectivity for a system consisting of a nonmagnetic substrate with a very thin cubic magnetic overlayer of thickness $d$, assuming that the sample is oriented with the $\{[100],[010],[001]\}$ axis parallel to the $\{x, y, z\}$ directions. The variation of the reflectivity up to first order in $d$ can be expressed as

$$
\begin{aligned}
\frac{\Delta R_{p p}}{R_{p p}}(\bar{M})= & A\left(\lambda, n_{a}, n_{f}, \theta\right) d \operatorname{Im}\left[\frac{a}{\varepsilon_{z z 0}}\right] M \sin (\omega t) \\
& +B\left(\lambda, n_{a}, n_{f}, \theta\right) d \operatorname{Im}\left[\frac{a^{2}}{\varepsilon_{z z 0}}+b_{11}+b_{12}\right. \\
& \left.+\left(-\frac{a^{2}}{\varepsilon_{z z 0}}+b_{11}-b_{12}\right) \cos (2 \omega t)\right] M^{2}
\end{aligned}
$$

where the coefficients $A$ and $B$ are given by

$$
\begin{gathered}
A\left(\lambda, n_{a}, n_{f}, \theta\right)=\frac{8 k_{0} n_{f} \cos \theta_{f} \sin \theta \cos \theta}{n_{f}^{2} \cos ^{2} \theta-n_{a}^{2} \cos ^{2} \theta_{f}}, \\
B\left(\lambda, n_{a}, n_{f}, \theta\right)=\frac{2 k_{0} n_{f} \cos ^{2} \theta_{f} \cos \theta}{n_{f}^{2} \cos ^{2} \theta-n_{a}^{2} \cos ^{2} \theta_{f}}
\end{gathered}
$$

where $\lambda$ is the wavelength of the light, $n_{a}$ and $n_{f}$ are the refractive indices of incident and substrate media, respectively, $\theta$ is the light incidence angle, and $\theta_{f}$ the light refraction angle. The first term corresponds to the variations of reflectivity, which have a linear dependence on the magnetization (term in $\omega$ ), and the second term corresponds to the variations, which have a quadratic dependence on the magnetization.

If the sample is rotated by $45^{\circ}$ around the $z$ axis we obtain

$$
\begin{aligned}
\frac{\Delta R_{p p}}{R_{p p}}(\bar{M})= & A\left(\lambda, n_{a}, n_{f}, \theta\right) d \operatorname{Im}\left[\frac{a}{\varepsilon_{z z 0}}\right] M \sin (\omega t) \\
& +B\left(\lambda, n_{a}, n_{f}, \theta\right) d \operatorname{Im}\left[\frac{a^{2}}{\varepsilon_{z z 0}}+b_{11}+b_{12}\right. \\
& \left.+\left(-\frac{a^{2}}{\varepsilon_{z z 0}}+2 b_{44}\right) \cos (2 \omega t)\right] M^{2} .
\end{aligned}
$$

It appears in Eqs. (8) and (10) that the quadratic term is proportional to constant coefficients and to coefficients that depend on the instant position of magnetization (term in $2 \omega$ ). These later coefficients change with measuring geometry [differences between Eqs. (8) and (10)] due to the magnetization-induced optical anisotropy. By extracting the variations of the reflectivity, which have an $\omega$ or $2 \omega$ dependence, the linear and quadratic magneto-optic coefficients of the magnetic layers, i.e., the off-diagonal and diagonal elements of the dielectric tensor, can be obtained. This analysis can be extended to layers of arbitrary thickness using a 4 $\times 4$ transfer-matrix formalism, ${ }^{20}$ which is used in the calculations hereafter.

\section{EXPERIMENTAL DETAILS}

Fe layers were grown on $\mathrm{MgO}(001)$ substrates in an ultrahigh vacuum sputtering plus laser ablation deposition system. Prior to the Fe deposition and in order to enhance the quality of the substrate, a 100 - $\AA$-thick $\mathrm{MgO}$ buffer layer was grown over the substrate at $450{ }^{\circ} \mathrm{C}$ by normal-incidence pulsed laser deposition in the pressure range of 7 $\times 10^{-9}$ mbar. The Fe films were deposited onto this $\mathrm{MgO}$ buffer layer by normal-incidence triode sputtering at 4 $\times 10^{-4}$ mbar Ar pressure and at deposition rates between 0.1 and $0.2 \AA /$ sec. Subsequently and to increase crystalline order, the samples were annealed at $400{ }^{\circ} \mathrm{C}$ for a few minutes. ${ }^{21}$ Finally and to prevent films from oxidation, a $30-\AA$-thick $\mathrm{MgO}$ capping layer was deposited at room temperature in similar conditions as for the previously described $\mathrm{MgO}$ buffer layer. In this study Fe layer nominal thicknesses ranged from 5 to $500 \AA$.

The magneto-optical experimental setup is depicted in Fig. 1. Light from a xenon lamp is $p$ polarized and focalized on the sample by a spherical mirror. The sample is located inside the bores of a $0.45-\mathrm{T}$ magnet, which rotates at $\omega$ frequency and forces the magnetization to turn in the plane of the sample. After reflection on the sample, the light is $p$ polarized and focalized into a monochromator. The resulting monochromatic light is then detected by a photodiode and the signal is processed with a lock-in amplifier. When set to the frequency $\omega$, the lock-in amplifier detects the variations of the reflectivity, which have a linear dependence with the magnetization, whereas the quadratic-dependent variations are given by the second harmonic. 

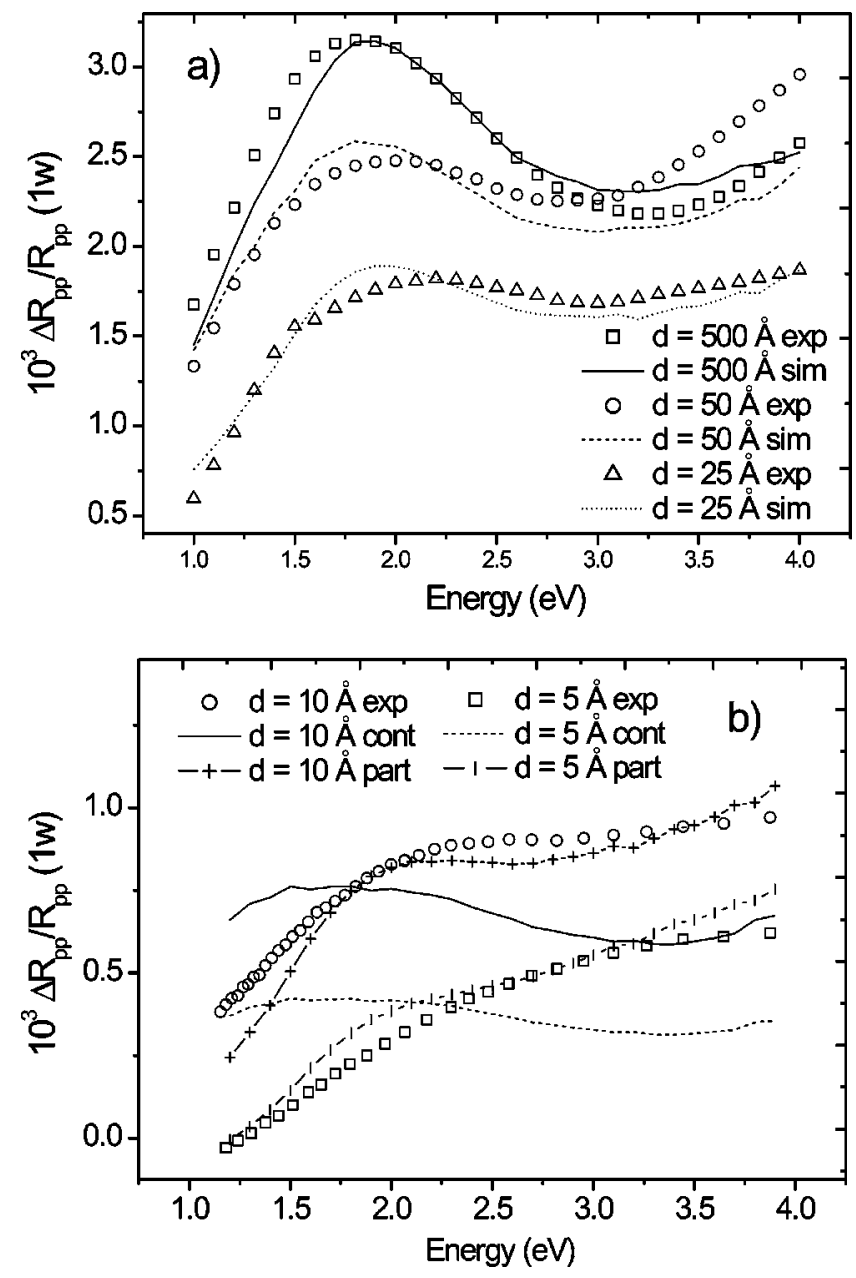

FIG. 2. (a) Measured linear magnetization variations of reflectivity (symbols) and corresponding calculated spectra (continuous and dashed lines) for different Fe thicknesses (25, 50, and $500 \AA$ ); (b) same as (a) for the thinnest Fe films (5 and $10 \AA$ ) together with calculations assuming either continuous films or nanoparticles.

\section{EXPERIMENTAL RESULTS AND SIMULATIONS}

\section{A. Linear magnetization variations of reflectivity}

In Fig. 2 we present the measured relative variations of the reflectivity, which are linearly dependent on the magnetization [linear magnetization variations of reflectivity (LMVR)] for $p$ - $p$-polarized light as a function of the photon energy and for samples with different Fe thicknesses. The spectra were obtained at an angle of incidence of $45^{\circ}$. The spectra show a peak located at about $2 \mathrm{eV}$ whose intensity and shape decreases and expands, respectively, with decreasing $\mathrm{Fe}$ thickness. A rotation of the samples along the $z$ direction does not change the spectra, as theoretically expected from Eqs. (8) and (10). The corresponding calculated spectra are represented by lines (continuous, dashed, and marked). The simulations were performed using the $4 \times 4$ transfermatrix formalism and assuming continuous layers with the optical and magneto-optical constants of bulk Fe given in Ref. 22. For the thickest samples [Fig. 2(a)] there is good agreement between theoretical calculations and experimental results. On the other hand, for layers of nominal thickness of
5 and $10 \AA$ [Fig. 2(b)], there are significant differences between experimental results and simulations. These differences could be associated with changes in the electronic structure of Fe due to confinement effects, but the disagreement could also be attributed to a different morphology in these ultrathin films. In fact, it is well established that, for small coverages, the growth of $\mathrm{Fe}$ at room temperature followed by annealing leads to the formation of crystalline islands with the same crystallographic symmetry of continuous monocrystalline thick films. ${ }^{12-14,23}$ This is particularly evident when measuring hysteresis loops in the transverse configuration. These hysteresis loops have been measured with the magnetic field parallel to the easy magnetization direction of iron [100] and with the magnetic field parallel to the direction [110]. It has been observed that the crystallographic magnetic anisotropy (expected for a continuous monocrystalline iron film) detected for the thickest samples (25-, 50-, and $500-\AA$ Fe thick layers) is not present in the 5 - and $10-\AA \mathrm{Fe}$ films, in agreement with hysteresis loops obtained by Cebollada et $a l .{ }^{24}$ and Jordan et al. ${ }^{10}$ in continuous and particulate systems. Moreover, the saturation field for these films is higher than the ones for the thicker films. Therefore such changes in the magnetic behavior are consistent with a modification of the morphology of the films: Fe films start to grow as islands (where shape anisotropy plus dipolar interactions between islands will hide their intrinsic crystallographic magnetic anisotropy and will have higher coercitivity fields) that coalesce with increasing $\mathrm{Fe}$ and form continuous films at higher $\mathrm{Fe}$ coverage. ${ }^{12,14}$ We have therefore used a selfconsistent effective-medium approach ${ }^{25-28}$ to study the impact of the morphology on the magneto-optical properties. This formalism has been already successfully used for the theoretical treatment of inhomogeneous materials and composites. ${ }^{17,27,29}$ For simplicity in the calculations these islands have been assumed to be magnetic ellipsoidal-shaped nanoparticles, which is coherent with atomic force microscopy ${ }^{23}$ and scanning tunneling microscopy observations. ${ }^{10}$ Within this model, the optical properties of the inhomogeneous medium are described by an effective dielectric tensor $\varepsilon_{e i j}$. If the matrix material $(\mathrm{MgO})$ is isotropic and nanoparticles keep the same crystallographic symmetry as that for continuous layers, the effective dielectric tensor has the same symmetry of the dielectric tensor of a homogeneous magnetic material, and the same number of magneto-optic coefficients will be needed to describe the magneto-optic properties of the system (see the Appendix).

An estimation of the size and shape of the particles can be obtained from the magnetization reversal loops in polar and transversal configurations if it is assumed that the system is superparamagnetic. Such an assumption is consistent with the absence of hysteresis in the 5- $\AA$ layer magnetization reversal loop and with magnetic observations of Adenwalla et $a l .{ }^{9}$ In the superparamagnetic fit, the shape anisotropy energy of the particles is introduced through the demagnetizing factors assuming that the particles are ellipsoids with revolution symmetry in the $z$ axis. The sizes of the particles obtained from these fits are 3.2 and $7.5 \mathrm{~nm}$ for a long semiaxis (in the $x y$ plane) and $1.5 \mathrm{~nm}$ for a short semiaxis (in the $z$ direction), for samples of 5 and $10 \AA$, respectively (although 
the thicker layer is already ferromagnetic). With these sizes, the effective dielectric tensor of the layer depends only on the concentration of the Fe nanoparticles. The calculations have been performed using the optical and magneto-optical constants of bulk iron for the nanoparticles. The best fits of the experimental spectra [given in Fig. 2(b)] were obtained for volumic concentrations of $10 \%$ and $20 \%$ of particles for the samples of 5- and 10- $\AA$ nominal Fe thicknesses, respectively. The agreement of such theoretical fits with the experimental results supports the nanoparticulate nature of the structure for these low Fe coverages. The thickness of the inhomogeneous layer is set to the height of the islands estimated from superparamagnetic fits, i.e., $30 \AA$. Although these parameters give amounts of $\mathrm{Fe}$ slightly smaller than those expected from the nominal thickness, we can conclude that the differences between experimental results and simulations assuming continuous $\mathrm{Fe}$ films for the thinnest samples are mainly due to morphology effects in these ultrathin films.

\section{B. Quadratic magnetization variations of reflectivity in continuous films}

We now turn to the relative variations of the reflectivity, which have a quadratic dependence on magnetization [quadratic magnetization variations of reflectivity (QMVR)]. The QMVR for a $p$ - $p$-polarized light as a function of the photon energy and for the different $\mathrm{Fe}$ continuous films are represented in Fig. 3. The spectra were obtained at an angle of incidence of $45^{\circ}$. As expected from the analysis given in Sec. II, different spectra are obtained when the sample is oriented with the $[100]$ axis perpendicular to the plane of incidence [Fig. 3(a)] or with the [110] axis perpendicular to that plane [Fig. 3(b)] in the case of continuous Fe films. Such a difference is indicative of the presence of a strong magneto-optical anisotropy in the QMVR of this cubic system. In opposition to the LMVR case, the QMVR is weaker for the $500-\AA \mathrm{Fe}$ layer than for 50 - and $25-\AA$ Fe layers in both crystallographic orientations. This behavior is caused by an optical effect due to the high contrast of dielectric constants between the magnetic layer and the substrate and capping layers. The difference between the spectra in the two different crystallographic orientations is also greater for the 50 - and $25-\AA \mathrm{Fe}$ layers than for the 500- $\AA \mathrm{Fe}$ layer, an effect that will be explained in Sec. V. To simulate the spectra, knowledge of the wavelength dependence of $b_{11}-b_{12}$ and $b_{44}$ elements is necessary. However, as far as we know, such information has not been published yet. We have therefore extracted these elements from the spectra of the 500- and $50-\AA$ Fe layers. The results are presented in Fig. 4. These coefficients exhibit a narrow peak around $1.5 \mathrm{eV}$, another broader structure located at $2.5 \mathrm{eV}$, and the biggest quadratic contribution in modulus is located at $1 \mathrm{eV}$. This is consistent with theoretical calculations based on the iron band structure, which have predicted that the strongest quadratic magnetization contribution in diagonal elements of a dielectric tensor should be around $1 \mathrm{eV}^{30}$ To check the validity of the coefficients we have simulated the QMVR spectra for a sample with thickness of $200 \AA$, obtaining good agreement with the experi-
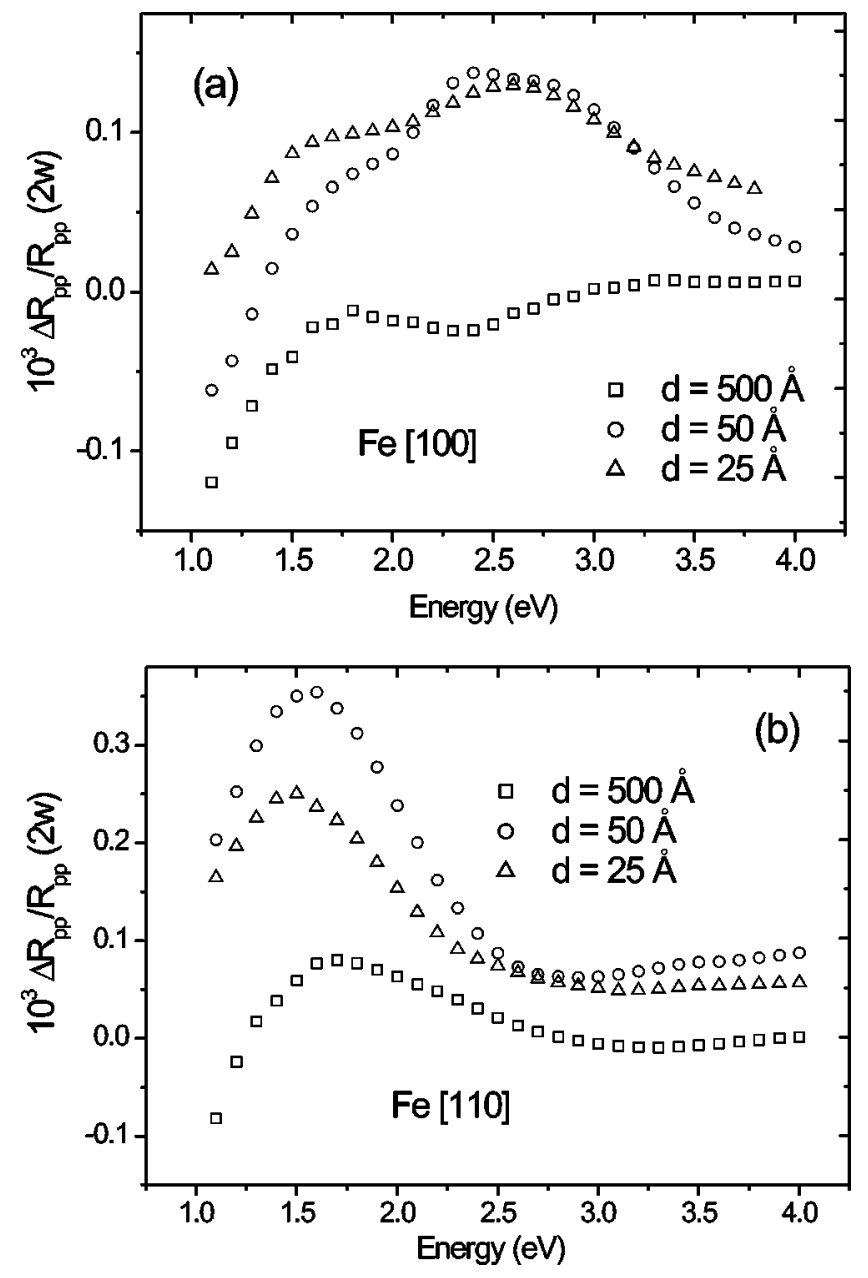

FIG. 3. (a) and (b) Measured quadratic magnetization variations of reflectivity for thickest Fe films $(25,50$, and $500 \AA$ ); (a) [100] axis perpendicular to the plane of incidence; (b) [110] axis perpendicular to the plane of incidence.

mental results. Moreover, a simulation QMRV for bulk iron at an angle of incidence of $10^{\circ}$ fits the experimental results obtained by Krinchik et al. ${ }^{31}$ in the corresponding photon energy range.

\section{QMVR in discontinuous films}

In Figs. 5(a) and 5(b) we present the QMVR experimental results for the layers of 5 and $10-\AA$ nominal thicknesses together with theoretical simulations assuming continuous layers and Fe nanoparticle layers and using the $b_{11}-b_{12}$ and $b_{44}$ elements determined for continuous films in the previous section. As can be observed, there is a strong deviation of the experimental results from theoretical calculations when the Fe deposits are assumed to be continuous layers. The shapes of the spectra change and there is an attenuation in the magnetization-induced optical anisotropy that was very strong in continuous films. The disagreement between the simulated spectra assuming two-dimensional growth and the experimental data is even greater for the QMVR than for the LMVR, suggesting a much higher sensitivity to the morphology for the QMVR than for the LMVR. On the other hand, 

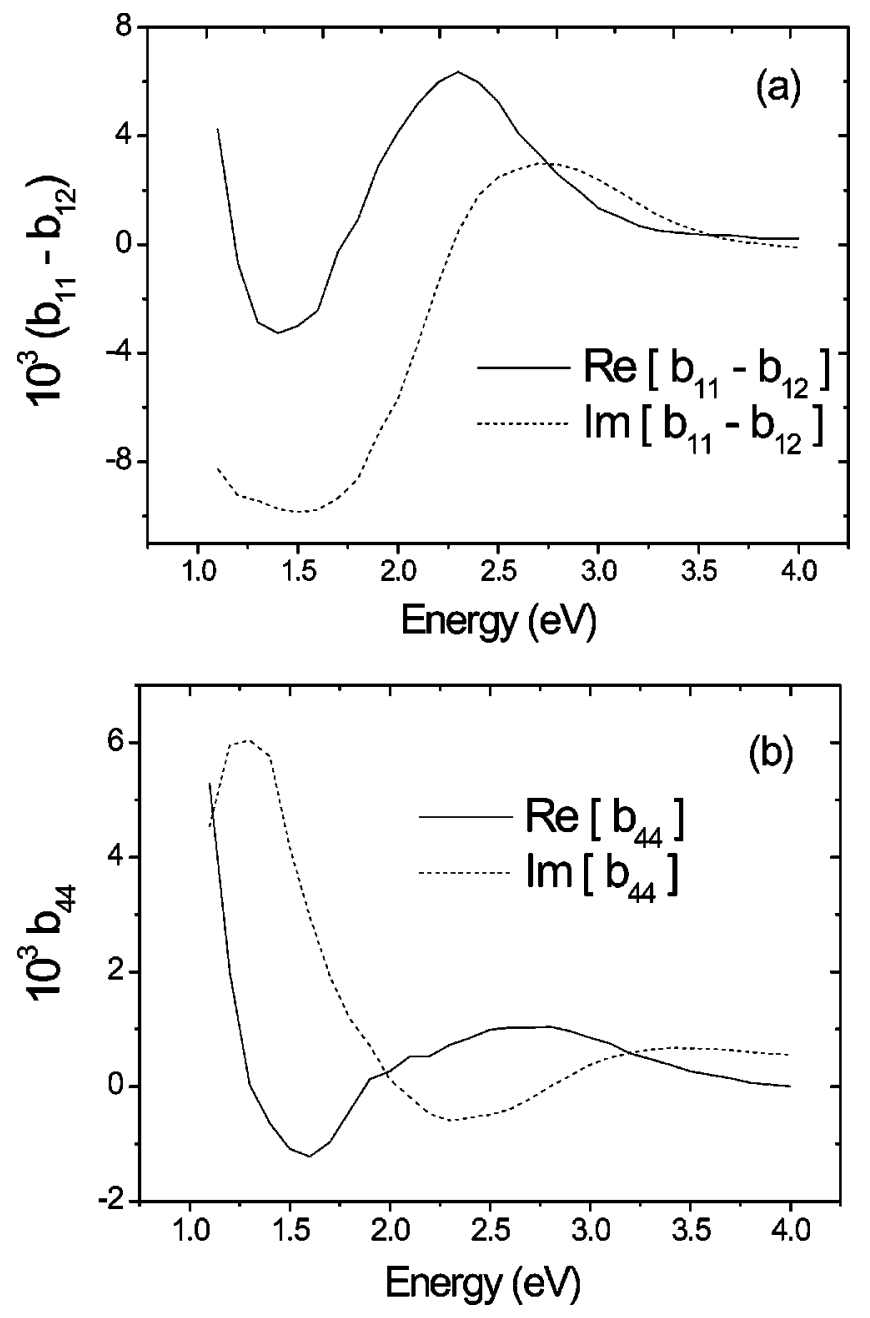

FIG. 4. (a) Photon energy evolution of the $b_{11}-b_{12}$ magnetooptical coefficient; (b) same for the $b_{44}$ coefficient.

simulations substituting the continuous Fe layer by an effective medium using the same model and parameters as the ones used in LMVR simulations present very good agreement, confirming that the morphology plays a very important role in the QMVR spectra.

\section{DISCUSSION}

As we have shown, the QMVR are more sensitive to the morphology than are LMVR. Besides, we have observed a strong reduction of the magnetization-induced optical anisotropy in the samples formed by nanoparticles with respect to the continuous films. The reduction of this magneto-opticalinduced optical anisotropy may be either due to a random distribution of the crystallographic axes of the iron islands, or to a shape effect. According to reflection high-energy electron-diffraction measurements the particles and the continuous layers have the same axes orientation, therefore, the reduction of anisotropy should be related to a shape effect. To understand its origin we study the different components of the calculated effective dielectric tensor, which contribute to QMVR measurements for an inhomogeneous layer. As is shown in the Appendix (Sec. 1), to describe the optical prop-
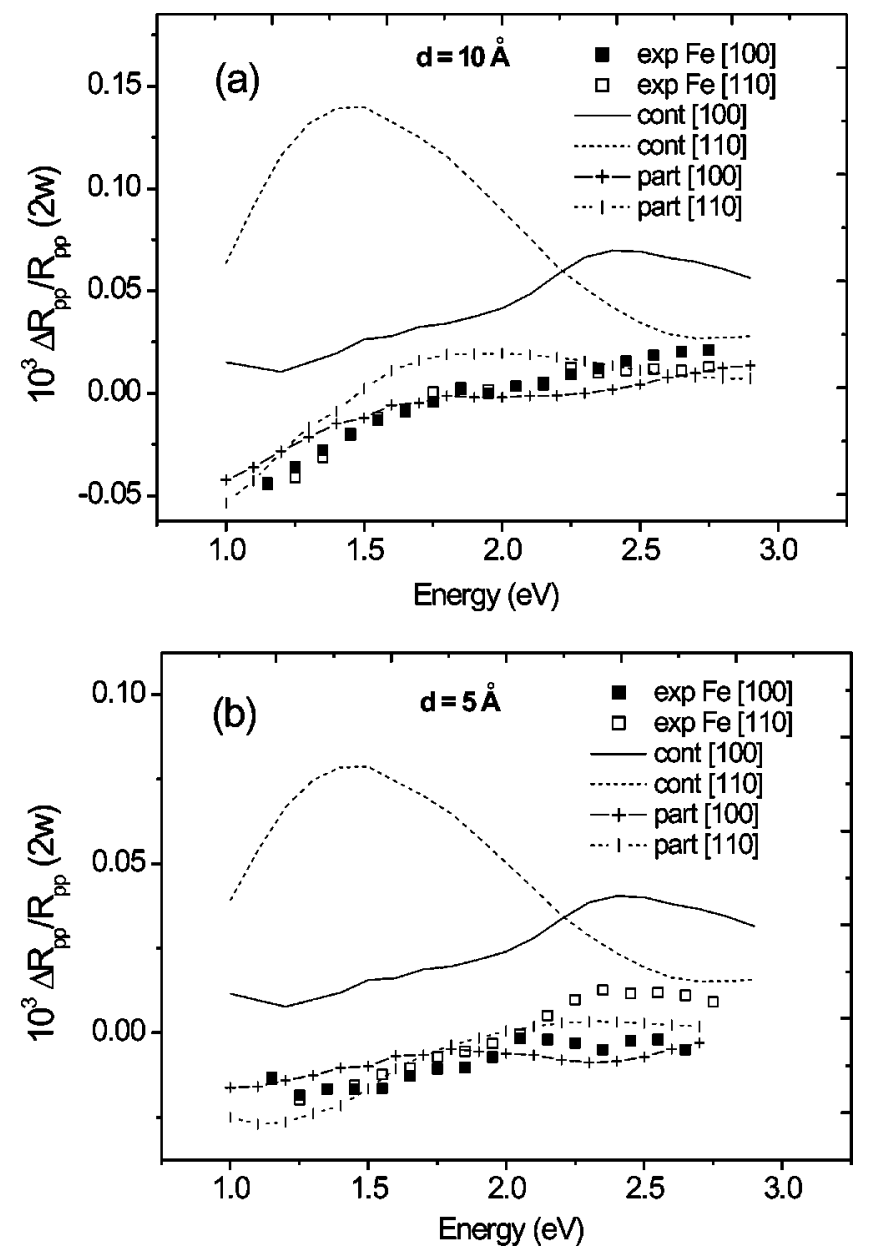

FIG. 5. (a) and (b) Same as Fig. 3 for the thinnest Fe films (5 and $10 \AA$ ) together with calculations assuming either continuous films or nanoparticles.

erties of our inhomogeneous media it is necessary to have the same number of magneto-optic coefficients as that for homogeneous media. Therefore we call $a_{e}$ the effective linear magneto-optic coefficient and $b_{e 11}, b_{e 12}$, and $b_{e 44}$ the effective quadratic magneto-optic coefficients. Looking at Eqs. (8) and (10), the terms that contribute to QMVR are $a_{e}^{2} / \varepsilon_{e z z 0}$, which is an isotropic term, and $\left(b_{e 11}, b_{e 12}\right)$ and $b_{e 44}$, which take into account the anisotropy induced by the crystalline order of the nanoparticles. Nevertheless, it is shown in the Appendix (Sec. 3) that there is a shape-induced quadratic effect described by the quadratic magneto-optic coefficient $b_{n s}$. Such a shape-induced quadratic magneto-optic coefficient $b_{n s}$ is found through the effective-medium formalism ignoring the quadratic coefficients of the magnetic material [Eqs. (A11)-(A16) in the Appendix]. This shapeinduced term is isotropic [see the Appendix, Eqs. (A17)(A19)], unlike the effective quadratic crystallographic terms $\left(b_{e 11}, b_{e 12}\right.$, and $\left.b_{e 44}\right)$. In Fig. 6 we present the wavelength dependence of these calculated quadratic terms, assuming an inhomogeneous layer containing a $10 \%$ volumic concentration of particles. As can be observed, the isotropic term $a_{e}^{2} / \varepsilon_{e z z 0}$ is very small compared to the shape-induced magneto-optical coefficient $b_{n s}$ and to $b_{e 11}, b_{e 12}$, and $b_{e 44}$. 

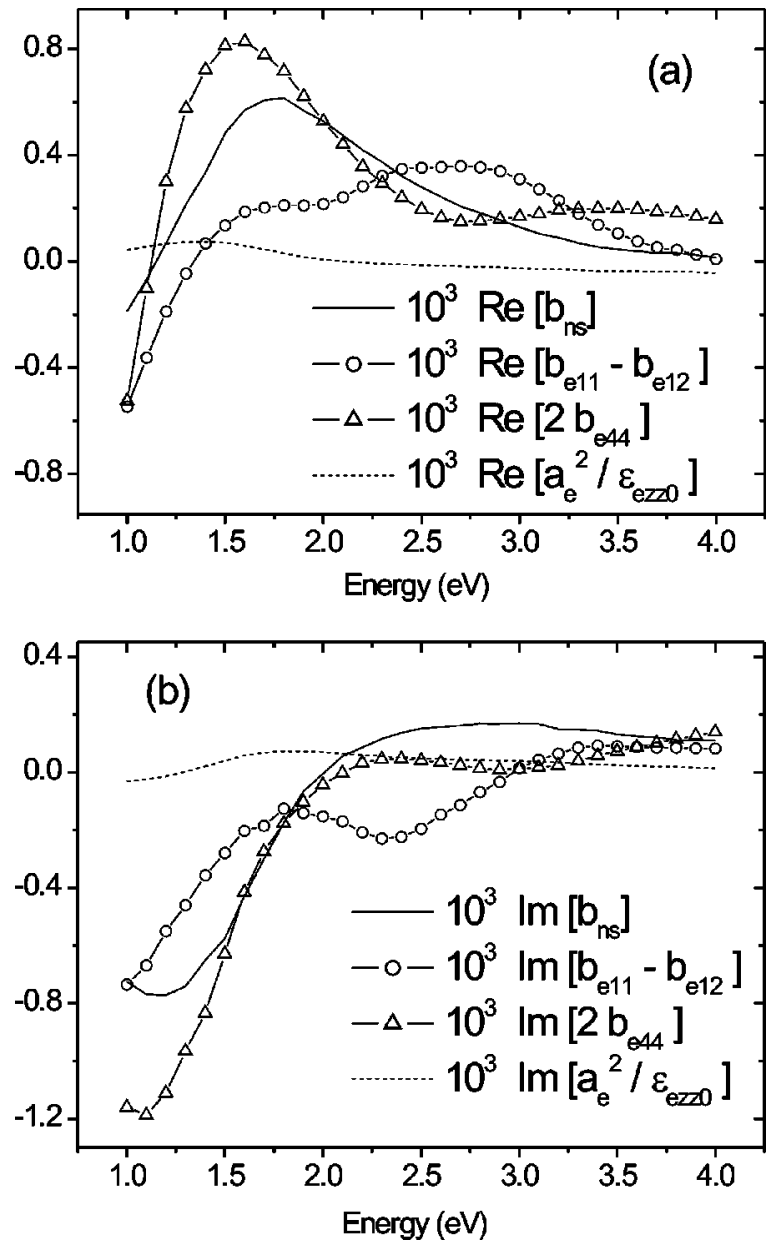

FIG. 6. Real and imaginary parts of the effective dielectric tensor components that are relevant for QMVR anisotropy. The assumed volumic concentration of the particles is $10 \%$.

Figure 6 shows as well that the shape-induced term has the same magnitude as the effective crystallographic terms and, as a consequence, the introduction of the quadratic contribution of the magnetic material produces only small differences from the shape-induced spectrum. In conclusion, the shapeinduced effect is responsible for the attenuation of magnetization-induced optical anisotropy.

As we increase the volumic concentration of particles in the layer we expect an increase in the magneto-optical anisotropy. In Fig. 7 we present the evolution of magnetizationinduced anisotropy as a function of nanoparticle concentration. Since an isotropic material is characterized by the relation presented in Eq. (4), in order to quantify the anisotropy, we have calculated the area enclosed between the spectra of real and imaginary parts of effective quadratic magneto-optic coefficients $\left(b_{e 11}-b_{e 12}\right)$ and $2 b_{e 44}$ for different concentrations:

$$
\begin{aligned}
& \operatorname{Re} b_{\text {anis }}=\int_{E_{1}}^{E_{2}}\left|\operatorname{Re}\left[\left(b_{e 11}-b_{e 12}\right)-2 b_{e 44}\right]\right| d E, \\
& \operatorname{Im} b_{\text {anis }}=\int_{E_{1}}^{E_{2}}\left|\operatorname{Im}\left[\left(b_{e 11}-b_{e 12}\right)-2 b_{e 44}\right]\right| d E,
\end{aligned}
$$

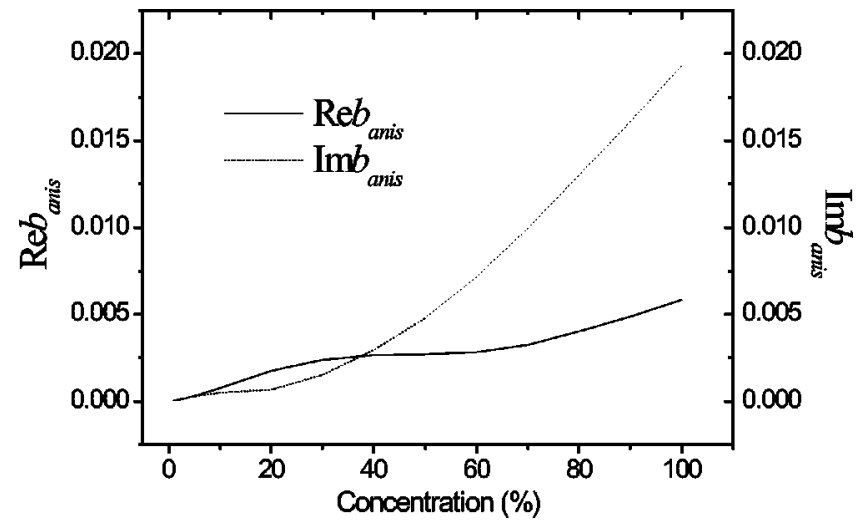

FIG. 7. Evolution of the magnetization-induced anisotropy in the real and imaginary parts of quadratic magneto-optic coefficients $\left(b_{\text {anis }}\right)$ with Fe concentration.

where $E 1$ and $E 2$ are the initial and final energies of 1 and 4 $\mathrm{eV}$, respectively. As can be observed, the evolution of anisotropy of real and imaginary parts of quadratic magneto-optic coefficients is not a linear function of the amount of Fe. In addition, there is a strong reduction of anisotropy in the imaginary part of diagonal dielectric elements, while this reduction is weaker in the real part, when concentration decreases. This different behavior of the real and imaginary parts of quadratic magneto-optic coefficients has important consequences in the evolution of the magneto-optical anisotropy in QMVR measurements as a function of the layer thickness. In Fig. 8 we present the anisotropy in QMVR for different layer thicknesses as a function of particle volumic concentration. This anisotropy is given by the area enclosed between the spectra of QMVR in the two different crystallographic orientations studied,

$$
\mathrm{QMVR}_{\text {anis }}=\int_{E_{1}}^{E_{2}}\left|\mathrm{QMVR}_{100}-\mathrm{QMVR}_{110}\right| d E .
$$

For the thinner layers the anisotropy presents a monotonic increase with nanoparticle concentration. Equations (8) and

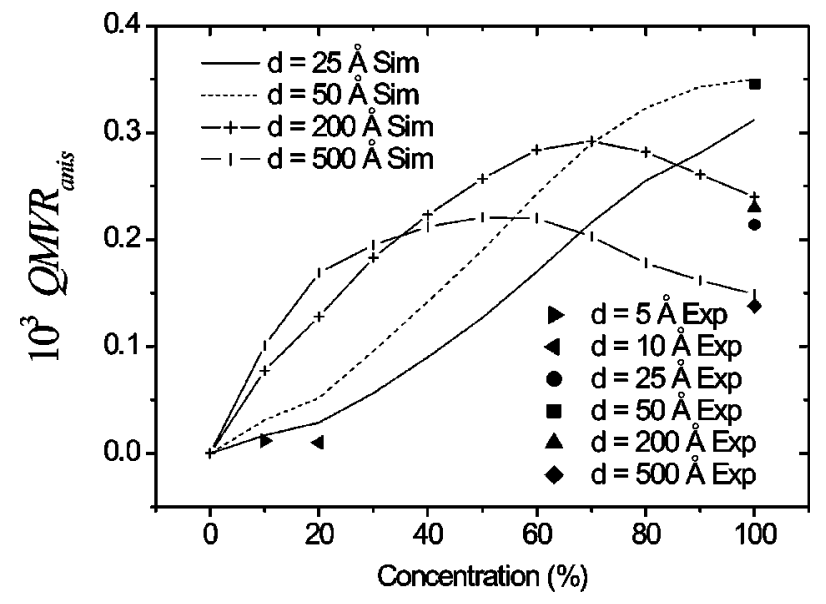

FIG. 8. Experimental and theoretical evolution of the anisotropy in QMVR $\left(\mathrm{QMVR}_{\mathrm{anis}}\right)$ for different Fe thicknesses and concentrations. 
(10) show that in very thin layers QMVR depend mainly on the imaginary part of the quadratic magneto-optic coefficients and, therefore, the concentration dependence of anisotropy is similar to the imaginary part of Fig. 7, as can be observed by comparing the shape of $\operatorname{Im} b_{\text {anis }}$ in Fig. 7 and $\mathrm{QMVR}_{\text {anis }}$ for 25- and 50- $\AA$ layers in Fig. 8. On the other hand, for thicker layers, it can be seen that anisotropy presents a maximum that decreases with increasing concentration. This is due to the dependence of the QMVR on both real and imaginary parts of quadratic magneto-optic coefficients for thick layers. This results in the mixing of the shape of the spectra of $\operatorname{Re} b_{\text {anis }}$ and $\operatorname{Im} b_{\text {anis }}$ from Fig. 7. This behavior also explains the weaker anisotropy found in 200- and $500-\AA \mathrm{Fe}$ layers than that found in 25 - or $50-\AA \mathrm{Fe}$ layers.

In Fig. 8 we have also represented the experimentally observed anisotropy for the different layer thicknesses studied, showing a reasonable agreement with theoretical calculations, except for the $25-\AA$ layer. In this layer the LMVR theoretical fit shows that this layer is continuous, however, the divergence in QMVR anisotropy can be attributed to the roughness of the layer. From Fig. 8 we have estimated that the volumic concentration of iron in this layer is around $60 \%$ (coalescence occurs approximately at 50\% volumic concentration) with a layer thickness of $40 \AA$. Such estimation is consistent as well with the growth mode described in Ref. 12 , confirming the sensitivity of QMVR to morphology changes.

Regarding the electronic structure of iron in discontinuous systems, previous studies of $\mathrm{Fe}$ nanoparticle systems have shown that Fe nanoparticles smaller than $4 \mathrm{~nm}$ present confinement effects that modify the electronic structure. ${ }^{32}$ Since our estimations give rise to bigger particles and providing that the calculations of the effective medium with optical and magneto-optical constants of bulk iron fit the experimental results, we are able to conclude that the $\mathrm{Fe}$ islands grown on $\mathrm{MgO}$ (001) keep the electronic structure of bulk iron.

\section{CONCLUSIONS}

We have presented the effect of morphology on the magneto-optic constants for the case of ultrathin monocrystalline iron films grown on $\mathrm{MgO}$ (001) substrates. The study is based on the behavior of linear and quadratic magnetooptic coefficients of iron, which have been probed through LMVR and QMVR measurements, showing that QMVR are more sensitive to morphology than are LMVR. Thick layers do not present morphology effects, allowing the extraction of the quadratic magneto-optic coefficients of monocrystalline iron from their QMVR measurements. These measurements have also evidenced the magnetization-induced optical anisotropy theoretically expected for monocrystalline iron films. In the case of very thin films we have shown that morphology plays a very important role in the behavior of magneto-optic coefficients. These layers are formed by islands of nanometric size with the same crystallographic axes orientation of the continuous films. The theoretical study of these systems has been carried out with a self-consistent effective-medium theory. Shape divergence of the experimental spectra from theoretical calculations of continuous layers and the attenuation in magnetization-induced anisotropy have been explained through this formalism. We show that the electronic structure of the grown nanometric Fe islands is similar to the bulk electronic structure and that the modifications of the magneto-optic coefficients are due to morphology effects.

\section{ACKNOWLEDGMENTS}

This work was performed under financial support of the Spanish Commission of Science and Technology. B. Sepúlveda acknowledges the I3P program of the Consejo Superior de Investigaciones Científicas (CSIC) and Y. Huttel acknowledges CSIC for financial support.

\section{APPENDIX}

First in this Appendix we discuss the magneto-optical constants needed to describe an inhomogeneous medium through an effective-medium formalism, when quadratic magneto-optic coefficients are introduced. Second we show that the nanostructuration itself induces a quadratic magnetooptic effect without taking into account the quadratic magneto-optic coefficients of iron and that this effect is isotropic.

\section{Self-consistent approximation}

One method to describe the optical properties of an inhomogeneous medium formed by nanoparticles embedded in a matrix is the self-consistent approximation. ${ }^{25-28}$ Within this approximation, inhomogeneous media are described by an effective dielectric tensor, which is calculated through the resolution of the matrix equation

$$
\left\langle\sum_{i} f_{i}\left(\overline{1}+\overline{\delta \varepsilon_{i}} \cdot \overline{\Gamma_{i}}\right)^{-1} \overline{\delta \varepsilon_{i}}\right\rangle=0
$$

where matter inside the brackets denotes volume average. If all the nanoparticles have the same shape, and present the same orientation in space, Eq. (A1) becomes

$$
f\left(\overline{1}+\overline{\delta \varepsilon_{p}} \cdot \overline{\Gamma_{p}}\right)^{-1} \overline{\delta \varepsilon_{p}}+(1-f)\left(\overline{1}+\overline{\delta \varepsilon_{m}} \cdot \overline{\Gamma_{m}}\right)^{-1} \overline{\delta \varepsilon_{m}}=0
$$

where

$$
\begin{aligned}
& \overline{\delta \varepsilon_{p}}=\overline{\varepsilon_{p}}-\overline{\varepsilon_{e}}, \\
& \overline{\delta \varepsilon_{m}}=\overline{\varepsilon_{m}}-\overline{\varepsilon_{e}},
\end{aligned}
$$

with $\varepsilon_{p}$ being the dielectric tensor of the particles, $\varepsilon_{m}$ the dielectric tensor of the matrix, and $\varepsilon_{e}$ the effective dielectric tensor; $\Gamma_{p}$ and $\Gamma_{m}$ are the depolarization tensors of the particles and matrix, respectively, 1 stands for the identity 3 $\times 3$ tensor, and $f$ is the volumic concentration of nanoparticles. Within this approximation the concentration can range from 0 to $100 \%$ of particles, providing the size of the particles is much smaller than the light wavelength. It is assumed in this formalism that the matrix is formed by spherical particles. If nanoparticles are ellipsoids with revolution 
symmetry around the $z$ axis, analytical expressions for the depolarization tensors can be found. ${ }^{28}$

$$
\begin{gathered}
\Gamma_{j x x}=\Gamma_{j y y}=-\frac{1}{2 \varepsilon_{e x x}}\left[\frac{1}{e_{j}^{2}}-\frac{\left(1-e_{j}^{2}\right)}{e_{j}^{3}} \tanh ^{-1} e_{j}\right], \\
\Gamma_{j z z}=-\frac{1}{\varepsilon_{e z z}}\left[-\frac{\left(1-e_{j}^{2}\right)}{e_{j}^{2}}+\frac{\left(1-e_{j}^{2}\right)}{e_{j}^{3}} \tanh ^{-1} e_{j}\right],
\end{gathered}
$$

where $\Gamma_{j k l}=0$ for $k \neq l$.

$e_{j=p}$ (for the particles) and $e_{j=m}$ (for the matrix) are given by

$$
\begin{gathered}
e_{p}=\sqrt{1-\frac{a_{p}^{2} \varepsilon_{e z z}}{c_{p}^{2} \varepsilon_{e x x}}}, \\
e_{m}=\sqrt{1-\frac{\varepsilon_{e z z}}{\varepsilon_{e x x}}} .
\end{gathered}
$$

\section{Nanoparticles with magnetization in the $X Y$ plane}

In our case, the experimental data from which we extract the quadratic magneto-optic coefficients $\left(b_{11}-b_{12}\right)$ and $b_{44}$ of the cubic magnetic material are measured in a configuration in which the magnetization is rotating in the $X Y$ plane. To introduce the obtained quadratic magneto-optic coefficients in the effective-medium model we assume a relative magnetization:

$$
\bar{M}=\frac{1}{\sqrt{2}}(1, i, 0)
$$

i.e., a magnetization in the $X Y$ plane with components $x$ and $y$ of the same magnitude but $\pi / 2$ out of phase, to take into consideration that the magnetization is rotating in the plane of the sample. Then the dielectric tensor of the nanoparticles becomes

$$
\overline{\varepsilon_{p}}=\left(\begin{array}{ccc}
\varepsilon_{p x x}+\frac{b_{11}-b_{12}}{2} & i b_{44} & \frac{i a}{\sqrt{2}} \\
i b_{44} & \varepsilon_{p x x}-\frac{b_{11}-b_{12}}{2} & -\frac{a}{\sqrt{2}} \\
-\frac{i a}{\sqrt{2}} & \frac{a}{\sqrt{2}} & \varepsilon_{p x x}
\end{array}\right),
$$

assuming a reference system $\{x, y, z\}$ parallel to $\{[100],[010],[001]\}$ crystallographic directions. By rotating the crystallographic axes $45^{\circ}$ around the $z$ axis, the dielectric tensor is transformed into

$$
\overline{\varepsilon_{p}}=\left(\begin{array}{ccc}
\varepsilon_{p x x}+b_{44} & -i \frac{b_{11}-b_{12}}{2} & \frac{i a}{\sqrt{2}} \\
-i \frac{b_{11}-b_{12}}{2} & \varepsilon_{p x x}-b_{44} & -\frac{a}{\sqrt{2}} \\
-\frac{i a}{\sqrt{2}} & \frac{a}{\sqrt{2}} & \varepsilon_{p x x}
\end{array}\right) .
$$

As can be observed, the formalism for the two crystallographic orientations is the same by interchanging the quadratic magneto-optic coefficients. In the following we therefore develop the formalism only in the first case.

Considering these dielectric tensors together with the depolarization tensor of the particles, the effective dielectric tensor must be written as

$$
\overline{\varepsilon_{e}}=\left(\begin{array}{ccc}
\varepsilon_{e x x}+\frac{b_{e 11}-b_{e 12}}{2} & i b_{e 44} & \frac{i a_{e}}{\sqrt{2}} \\
i b_{e 44} & \varepsilon_{e x x 0}-\frac{b_{e 11}-b_{e 12}}{2} & -\frac{a_{e}}{\sqrt{2}} \\
-\frac{i a_{e}}{\sqrt{2}} & \frac{a_{e}}{\sqrt{2}} & \varepsilon_{e z z 0}
\end{array}\right),
$$

where $a_{e}$ is the effective linear magneto-optic coefficient, whereas $b_{e 11}, b_{e 12}$, and $b_{e 44}$ are the effective quadratic magneto-optic coefficients. The elements $\varepsilon_{e x x 0}$ and $\varepsilon_{e z z 0}$ are the effective dielectric constants without magnetization and are different as a consequence of the birefringence caused by the shape of the particles. Such dielectric constants must be previously calculated for the nanoparticle system without magnetization, obtaining a set of two independent equations with the two unknowns $\varepsilon_{e x x 0}$ and $\varepsilon_{e z z 0}$. The calculated values are introduced in the tensor of Eq. (A10). Then Eq. (A2) is solved for the three unknown variables $a_{e},\left(b_{e 11}-b_{e 12}\right)$, and $b_{e 44}$. As a consequence, we need the same number of magneto-optic coefficients to describe the nanoparticle effective medium.

\section{Isotropic quadratic effect}

Now it is shown that nanostructure induces a quadratic effect with magnetization and that this effect is isotropic. Such an effect is described by a shape-induced magnetooptic coefficient $b_{n s}$. For this purpose we consider the simple case of spherical crystalline particles with the same axes orientation, when magnetization is in the $x$ axis, i.e., $\mathbf{M}=(M, 0,0)$, without taking into account quadratic magneto-optic coefficients of the magnetic material. If reference axes are parallel to crystallographic directions $\{[100]$, [010], [001]\} ( $M$ parallel to the direction [100]), the dielectric tensor of the particles and the effective dielectric tensor are described by 


$$
\begin{gathered}
\varepsilon_{p}=\left(\begin{array}{ccc}
\varepsilon_{x x 0} & 0 & 0 \\
0 & \varepsilon_{x x 0} & -a \\
0 & a & \varepsilon_{x x 0}
\end{array}\right), \\
\varepsilon_{e}=\left(\begin{array}{ccc}
\varepsilon_{e x x}+b_{n s 11} & 0 & 0 \\
0 & \varepsilon_{e x x 0}+b_{n s 12} & -a_{e} \\
0 & a_{e} & \varepsilon_{e x x 0}+b_{n s 12}
\end{array}\right) .
\end{gathered}
$$

If reference axes are parallel to crystallographic directions $\{[110],[1-10],[001]\}$ ( $M$ parallel to the direction $[110])$, the dielectric tensor of the particles is the same as expression (A11), while the effective tensor is transformed into

$$
\varepsilon_{e}=\left(\begin{array}{ccc}
\varepsilon_{e x x 0}+\frac{b_{n s 11}+b_{n s 12}}{2}+b_{n s 44} & 0 & 0 \\
0 & \varepsilon_{e x x 0}+\frac{b_{n s 11}+b_{n s 12}}{2}-b_{n s 44} & -a_{e} \\
0 & a_{e} & \varepsilon_{\text {exx } 0}+b_{n s 12}
\end{array}\right) .
$$

Introducing the tensors of the first orientation in expression (A2), we obtain a set of three independent equations with three unknown variables $a_{e}, b_{n s 11}$, and $b_{n s 12}$ :

$$
\begin{gathered}
f \frac{\varepsilon_{x x 0}-\varepsilon_{e x x}-b_{n s 11}}{2 \varepsilon_{e x x}+\varepsilon_{x x 0}-b_{n s 11}}+(1-f) \frac{\varepsilon_{m}-\varepsilon_{e x x}-b_{n s 11}}{2 \varepsilon_{e x x}+\varepsilon_{m}-b_{n s 11}}=0 \\
f \frac{\left(a-a_{e}\right)^{2}+\left(\varepsilon_{x x 0}-\varepsilon_{e x x 0}-b_{n s 12}\right)\left(\varepsilon_{x x 0}+2 \varepsilon_{e x x}-b_{n s 12}\right)}{\left(a-a_{e}\right)^{2}+\left(\varepsilon_{x x 0}+2 \varepsilon_{e x x}-b_{n s 12}\right)^{2}}+(1-f) \frac{a_{e}^{2}+\left(\varepsilon_{m}-\varepsilon_{e x x}-b_{n s 12}\right)\left(\varepsilon_{m}+2 \varepsilon_{e x x 0}-b_{n s 12}\right)}{a_{e}^{2}+\left(\varepsilon_{m}+2 \varepsilon_{e x x 0}-b_{n s 12}\right)^{2}}=0 \\
f \frac{a_{e}-a}{\left(a-a_{e}\right)^{2}+\left(\varepsilon_{x x 0}+2 \varepsilon_{e x x}-b_{n s 12}\right)^{2}}+(1-f) \frac{a_{e}}{a_{e}^{2}+\left(\varepsilon_{m}+2 \varepsilon_{e x x 0}-b_{n s 12}\right)^{2}}=0 .
\end{gathered}
$$

The equations in the case of the second orientation of crystallographic axes are formally the same, but they interchange the effective quadratic coefficients. Therefore the following relations must be satisfied:

$$
\begin{aligned}
& b_{n s 11}=\frac{b_{n s 11}+b_{n s 12}}{2}+b_{n s 44}, \\
& b_{n s 12}=\frac{b_{n s 11}+b_{n s 12}}{2}-b_{n s 44},
\end{aligned}
$$

that is,

$$
\frac{b_{n s 11}-b_{n s 12}}{2}=b_{n s 44}
$$

showing that the effect is isotropic.

Since this effect is isotropic, we use only the first orientation to see its magnetization dependence. In Eq. (A14) the coefficient $b_{n s 11}$ is related only to terms that are not dependent on magnetization and, as a consequence, this term must be zero. On the other hand, Eqs. (A15) and (A16) form a set of two coupled equations with two unknowns, $a_{e}$ and $b_{n s 12}$. Solving these equations simultaneously it can be seen that both coefficients are magnetization dependent through the linear magneto-optic coefficient of iron, $a$. Setting the correct coefficients it is proved that the effective magneto-optic coefficient $a_{e}$ presents a linear dependence on magnetization, while the dependence is quadratic for $b_{n s 12}$. In conclusion, the nanostructure induces a quadratic effect, which is isotropic and is due to the coupling of the linear magneto-optic coefficient of the magnetic material $a$. Accordingly this effect can be described with only one coefficient, $b_{n s}$.

The introduction of quadratic magneto-optic coefficients of iron induces a magneto-optical anisotropy in the effective medium, since it has been proved in monocrystalline iron that

$$
\frac{b_{11}-b_{12}}{2} \neq b_{44}
$$

As a consequence, the effective quadratic coefficients must satisfy as well a later relation. By introducing the quadratic coefficients of the magnetic material in Eqs. (A14)-(A16) it can be observed that the effective coefficient $b_{e 11}$ is not zero and depends only on the quadratic coefficient of iron, $b_{11}$. Nevertheless the coefficient $b_{e 12}$ depends on both linear and 
quadratic coefficients of iron $\left(a\right.$ and $\left.b_{12}\right)$. Thus the quadratic effect of iron will be present together with the nanostructureinduced quadratic effect. Since we have shown that this effect is isotropic, it will tend to attenuate the anisotropy in- duced by the monocrystallinity of iron. In Fig. 6 it is observed that the nanostructure term is dominant for a $10 \%$ volumic concentration of particles, verifying the attenuation of anisotropy.
*Electronic address: borja@imm.cnm.csic.es

${ }^{1}$ N. B. Brookes, Y. Chang, and P. D. Johnson, Phys. Rev. Lett. 67, 354 (1991).

${ }^{2}$ J. E. Ortega, F. J. Himpsel, G. J. Mankey, and R. F. Willis, Phys. Rev. B 47, 1540 (1993).

${ }^{3}$ R. K. Kawakami, E. Rotenberg, H. J. Choi, E. J. EscoricaAparicio, M. O. Bowen, J. H. Wolfe, E. Arenholz, Z. D. Zhang, N. V. Smith, and Z. Q. Qiu, Nature (London) 398, 132 (1999).

${ }^{4}$ J. J. Pagel, T. Miller, and T. C. Chiang, J. Electron Spectrosc. Relat. Phenom. 101-103, 271 (1999).

${ }^{5}$ Y. Suzuki, T. Katayama, S. Yoshida, K. Tanaka, and K. Sato, Phys. Rev. Lett. 68, 3355 (1992).

${ }^{6}$ W. Geerts, Y. Suzuki, T. Katayama, K. Tanaka, K. Ando, and S. Yoshida, Phys. Rev. B 50, 12581 (1994).

${ }^{7}$ R. Megy, A. Bounouh, Y. Suzuki, P. Beauvillain, P. Bruno, C. Chappert, B. Lecuyer, and P. Veiller, Phys. Rev. B 51, 5586 (1995).

${ }^{8}$ A. Kirilyuk, P. E. Hansen, S. Yuasa, T. Katayama, and Th. Rasing, Surf. Sci. 402-404, 356 (1998).

${ }^{9}$ S. Adenwalla, Y. Park, G. P. Felcher, and M. Teitelman, J. Appl. Phys. 76, 6443 (1994).

${ }^{10}$ S. M. Jordan, J. F. Lawler, R. Schad, and H. Van Kempen, J. Appl. Phys. 84, 1499 (1998).

${ }^{11}$ G. Fashold, G. König, W. Theis, A. Lehmann, and K.-H. Rieder, Appl. Surf. Sci. 137, 224 (1999).

${ }^{12}$ G. Fashold, A. Lehmann, and K.-H. Rieder, Phys. Rev. B 61, 8475 (2000).

${ }^{13}$ G. Fashold, A. Priebe, and A. Pucci, Appl. Phys. A: Mater. Sci. Process. 73, 39 (2001).

${ }^{14}$ A. di Bona, C. Giovanardi, and S. Valeri, Surf. Sci. 498, 193 (2002).

${ }^{15}$ D. Weller, G. R. Harp, R. F. C. Farrow, A. Cebollada, and J. Sticht, Phys. Rev. Lett. 72, 2097 (1994).

${ }^{16}$ R. M. Osgood III, S. D. Bader, B. M. Clemens, R. L. White, and
H. Matsuyama, J. Magn. Magn. Mater. 182, 297 (1998).

${ }^{17}$ J. L. Menéndez, G. Armelles, A. Cebollada, C. Quintana, D. Ravelosona, C. Chappert, F. Peiró, and A. Cornet, Appl. Phys. Lett. 81, 1603 (2002).

${ }^{18}$ A. K. Zvezdin and V. A. Kotov, in Modern Magnetooptics and Magnetooptical Materials (Institute of Physics Publishing Bristol and Philadelphia, Bristol, 1997).

${ }^{19}$ J. F. Nye, in Physical Properties of Crystals (Oxford University Press, Oxford, 1985).

${ }^{20}$ M. Schubert, Phys. Rev. B 53, 4265 (1996).

${ }^{21}$ C. Martínez Boubeta, E. Navarro, A. Cebollada, F. Briones, F. Peiró, and A. Cornet, J. Cryst. Growth 226, 223 (2001).

${ }^{22}$ K. H. J. Buschow, in Ferromagnetic Material, edited by E. P. Wohlfarth and K. H. J. Buschow (Elsevier Science, Amsterdam, 1988).

${ }^{23}$ J. F. Lawler, R. Schad, S. Jordan, and H. van Kempen, J. Magn. Magn. Mater. 165, 224 (1997).

${ }^{24}$ F. Cebollada, A. Hernando-Mañeru, A. Hernando, C. MartínezBoubeta, A. Cebollada, and F. J. M. González, Phys Rev. B 66, 174410 (2002).

${ }^{25}$ D. Stroud, Phys. Rev. B 12, 3368 (1975).

${ }^{26}$ T. Xia, P. Hui, and D. Stroud, J. Appl. Phys. 67, 2736 (1990).

${ }^{27}$ T. Noah and P. Song, Phys. Rev. B 44, 5459 (1991).

${ }^{28}$ J. S. Ahn, K. H. Kim, and T. W. Noh, Phys. Rev. B 52, 15244 (1995).

${ }^{29}$ C.-Y. You, S.-C. Shin, and S.-Y. Kim, Phys. Rev. B 55, 5953 (1997).

${ }^{30}$ H. Ebert, Rep. Prog. Phys. 59, 1665 (1996).

${ }^{31}$ G. S. Krinchik, E. E. Chapurova, and S. E. Yurchenko, Fiz. Tverd. Tela (Leningrad) B6, 3096 (1973) [Sov. Phys. Solid State 15, 2043 (1974)].

32 J. L. Menendez, B. Bescós, G. Armelles, R. Serna, J. Gonzalo, R. Doole, A. K. Petford-Long, and M. I. Alonso, Phys. Rev. B 65 , 205413 (2002). 